\title{
Assessment of mitral valve function at operation and the prevention of systemic embolism
}

\author{
J. KEITH ROSS \\ National Heart Hospital, London
}

\begin{abstract}
When assessing the function of the mitral valve at operation by palpation or inspection, the surest way to prevent systemic (cerebral) embolism is to have the ascending aorta occluded by a clamp.

A technique is described which allows safe assessment of mitral valve function by aortic root and left ventricular perfusion, giving a beating-heart preparation with the aorta cross-clamped.

This method has been in successful use for more than two years and has been found particularly useful in the accurate repair of cleft mitral valves in atrioventricular defects (ostium primum defects and atrioventricular canal).
\end{abstract}

The problem of air embolism during intracardiac surgery and its prevention has been well reviewed recently by Tsuji, Redington, Mendez, and Kay (1970).

It has been the author's experience that the introduction of aortic cross-clamping and ischaemic arrest for intracardiac procedures has resulted in a major reduction in the incidence of postoperative cerebral complications. This in turn has led to mistrust of any situation in which the aorta is unclamped with the left heart chambers open to air and the heart beating.

The technique to be described offers a solution to the problems peculiar to assessment of the mitral valve with the heart open and beating.

Reservations about the safety of ischaemic arrest may be resolved by the use of aortic root perfusion alone, a simple technique which makes it possible to sustain the myocardium while maintaining the principle of aortic cross-clamping as the surest way of preventing cerebral emtolism.

\section{ASSESSMENT OF THE MITRAL VALVE}

Mitral valve function may need to be assessed at three different stages of an open operation. First, it may be planned to palpate the valve at the outset rather than proceeding to open the left atrium and fully expose the valve. Secondly, once the valve has been fully exposed, and if a conservative procedure is possible, then it may be desirable to see precisely what is wrong with the valve function before starting a repair or reconstruction. Thirdly, once the valve has been repaired, or replaced by a tissue valve, it is essen- tial to test the efficiency of the repair or replacement.

All three situations carry an embolic risk: in the first the hazard is left atrial thrombus and in the second and third it is air.

ASSESSMENT BY PALPATION Palpation of the mitral valve before establishing bypass has nothing to recommend it. If the exploring finger is put into the left atrium either before bypass is started or after starting perfusion but before clamping the aorta, then the risk of dislodging atrial clot is the same as for 'closed' mitral procedures. In other words, one of the great advantages of the open technique is cancelled out at the very beginning of the operation by digital invasion of the left atrium in the presence of an unclamped aorta.

Safe palpation of the mitral valve on bypass means that the digital assessment has to be made during the remaining vigorous systoles which happen after the aorta has been clamped. The fact that the force of ventricular contraction is lessening, and that the heart is becoming empty at the time, makes the value of the findings on palpation uncertain, and more valid for recognizing stenosis than for making an appraisal of regurgitation. In practice, it has not been found that these limitations matter a great deal: if there is any doubt about the findings on palpation in these rather artificial circumstances, it is our practice to expose the valve formally and to inspect it.

VALVe assessment Assuming that the aorta has been clamped before opening the left atrium, the 
heart is likely to be in arrest and becoming flaccid by the time the mitral valve is exposed. If the aortic clamp is now released (with or without tripping of the aortic valve to let blood into the left ventricle) the result is the dangerous combination of a beating heart with a mixture of blood and air in the left ventricle. Air may escape into the aorta if the mitral valve has even a little residual function, and the perfusion pressure cannot be relied upon to keep the aortic valve closed to the passage of air through it.

All the foregoing remarks apply equally to the assessment of valve function after repair or replacement.

The three requisites for safe and satisfactory assessment of mitral valve function under direct vision are:

1. a left ventricle filled with blood in a controlled fashion ;

2. a beating heart;

3. a clamp on the ascending aorta.

Technique The three requisites may be achieved by using the arrangement illustrated in the Figure.

Standard coronary perfusion cannulae are used, one introduced into the aorta via a purse string proximal to the clamp and the other into the apex of the left ventricle. It happens that the balloonended coronary cannula in use at the National Heart Hospital fits exactly inside the tubing used

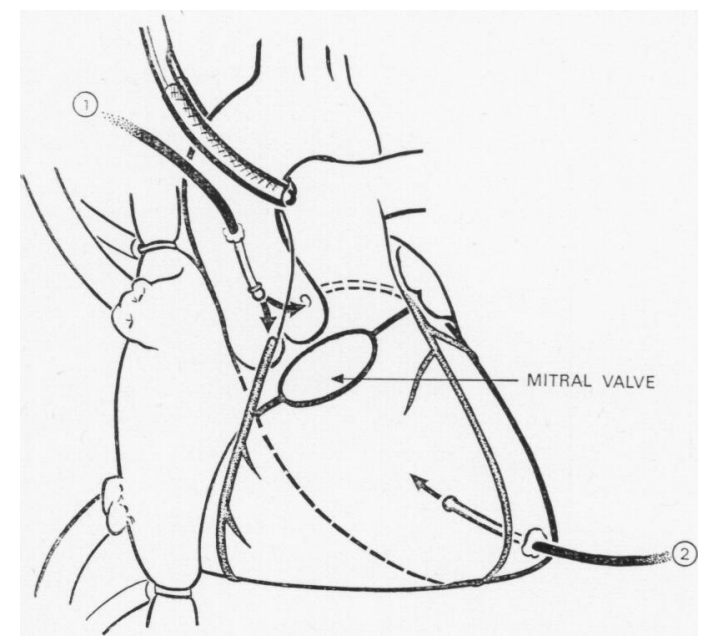

FIGURE. Method of assessment of mitral valve function. The aorta is clamped. Coronary cannula (1) is placed in the aortic root. Coronary cannula (2) is placed in the left ventricle. For simplicity, the position of the mitral valve is indicated diagrammatically. for the left ventricular vent, but there is no reason why the cannula should not be put through the ventricular wall direct, and this is in fact the routine practice with the right ventricle when assessing tricuspid function. The aortic root cannula is put in through a stab incision, and the purse string is not drawn tight until all air has been displaced from the aortic root by gently filling it with blood from the cannula.

Once the heart begins to beat, blood can be introduced in a controlled fashion into the left ventricle by judicious release of blood up the second cannula, avoiding over-distension of the ventricle. If this is done slowly most, if not all, of the air in the ventricle will be displaced before the valve shuts. The valve is then tripped as often as necessary to release blood from the ventricle when it becomes full, and the sequence is repeated as often as is required, allowing repeated assessment of valve function.

\section{DISCUSSION}

This technique does not guarantee that all air is displaced from the left ventricle and it might be expected to produce coronary air embolism in some instances. In practice, evidence that this has occurred has not been seen and it has not been a problem.

The method described has been in use for more than two years and has proved most valuable in assessing the results of all types of mitral valve reconstruction. Its most significant application has been in the management of the cleft mitral valve in atrioventricular defects (ostium primum and atrioventricular canal) when a 'graded' repair of the valve is possible, by repeated testing as the repair progresses, allowing optimal closure of the cleft. In this way, in addition to making sure that all regurgitation has been checked, unnecessary extra sutures are avoided, thereby lessening the risk of producing stenosis. This goes a long way towards ending the need for inspired guess-work when repairing these valves and introduces precision into an otherwise rather intuitive procedure.

The technique does depend upon the presence of a competent aortic valve. Minor degrees of aortic regurgitation, sometimes caused by distortion by retractors, have not been found troublesome either from the point of view of effective coronary perfusion or of visualization of the mitral valve.

Aortic root cannulation and perfusion, alone, has been used successfully on occasions when it has been felt that the safe limit of ischaemic arrest 
has been reached and the operation on the mitral valve has not been finished. As already stated, this can also be used electively from the outset when there is doubt about the safety of using anoxic arrest.

Use of the left ventricular cannula, alone, to test the mitral valve with the heart in ischaemic arrest has also been extensively used without complication. Here, the risk of over-distension of the flaccid left ventricle is very real and must be avoided. Although the assessment of the mitral valve made in this way is reliable (and entirely satisfactory if the valve has been replaced, for example by a tissue valve) when the subvalve mechanism is intact there is much to be said for having the heart beating when the result of valve reconstruction has to be judged.

Finally, the aortic root cannula may be divided at the end of the procedure, when the left heart has been closed, and its end directed up a sucker as a convenient vent for any residual air at the time of release of the aortic clamp.

\section{REFERENCE}

Tsuji, H. K., Redington, J. V., Mendez, A., and Kay, J. H. (1970). The prevention of air embolism during intracardiac surgery. J. thorac. cardiovasc. Surg., 59, 484. 